\title{
Large number of phosphotransferase genes in the Clostridium beijerinckii NCIMB 8052 genome and the study on their evolution
}

\author{
Yixiang Shi ${ }^{1,2}$, Yi-Xue $L i^{1,2}$, Yuan-Yuan $L i^{1,2^{*}}$ \\ From The $21^{\text {st }}$ International Conference on Genome Informatics (GIW2010) \\ Hangzhou, People's Republic of China. 16-18 December 2010
}

\begin{abstract}
Background: Clostridium beijerinckii is a valuable bacteria species which has the ability of ABE (acetone, butanol and ethanol) production. It has been shown that Phosphotransferase (PTS) is an important and common system for both carbohydrate uptake and phosphorylation in bacteria, but detailed study of the system, especially its fructose/mannose/sorbose family is scant.

Results: In the genome of Clostridium beijerinckii NCIMB 8052, a model strain recently sequenced, there are large number of PTS genes, among them 9 complete sets belong to the fructose/mannose/sorbose family of its enzyme II complex. Our study, based on evidences provided by phylogenetic relationship, analyses of gene contents and clusters, as well as synteny examination, indicates that it is possible to further classify this PTS family into three subgroups, which are corresponding to the three sugar substrates. Furthermore, we proposed a model how these PTS systems are evolved in bacteria.

Conclusion: This work may explain the experimental result that Clostridium beijerinckii NCIMB 8052 can better utilize fructose as substrate, thus could lead to a better understanding of the ABE-producing mechanism in Clostridium beijerinckii and other microbial species. It may help to illustrate a higher butanol-productivity future.
\end{abstract}

\section{Background}

The anaerobic Gram-positive bacterium Clostridium beijerinckii is capable of producing acetone, butanol and ethanol (which are together called ABE) [1]. These three are important chemical products, especially butanol, since it is not only a food-grade solvent, but also an excellent fuel potentially could be used as a replacement for oil. Its features such as high boiling point and low emission are quite desirable [1]. Similar to another ABE-producing model strain Clostridium acetobutylicum ATCC 824, the ratio of the solvent produced by Clostridium beijerinckii is close to 3:6:1 (A:B:E, v/v/v) [1,2]. But Clostridium beijerinckii has the advantage that its

\footnotetext{
* Correspondence: yyli@scbit.org

1 Bioinformatics Center, Key Laboratory of Systems Biology, Shanghai Institutes for Biological Sciences, Chinese Academy of Sciences, Shanghai 200031, P.R. China

Full list of author information is available at the end of the article
}

fermentation does not require the using of starch, which is a food source. It can utilize various kinds of biomass, including 'waste' material, therefore can lower the producing cost and enhance its practical competitiveness $[1,3]$. Because of its great industrial potential, its model strain Clostridium beijerinckii NCIMB 8052 has been sequenced by JGI (Joint Genome Institute, DOE, USA) and the genomic data were made public in 2007 (http:// genome.jgi-psf.org/clobe/clobe.home.html). The ABEproducing process starts from pyruvate, the end product of glycolysis. Pyruvate can be further degraded into acetyl-CoA. Acetyl-CoA could be transformed into ethanol, or through several steps, into butanol and acetone (see Figure 1 of the reference [4]). So, the more monosaccharide molecules which are transferred into cells, and the higher sugar utilization efficiency, the better for the ABE-production. Phosphotransferase system (PTS) is an important mechanism for sugar transmembrane 


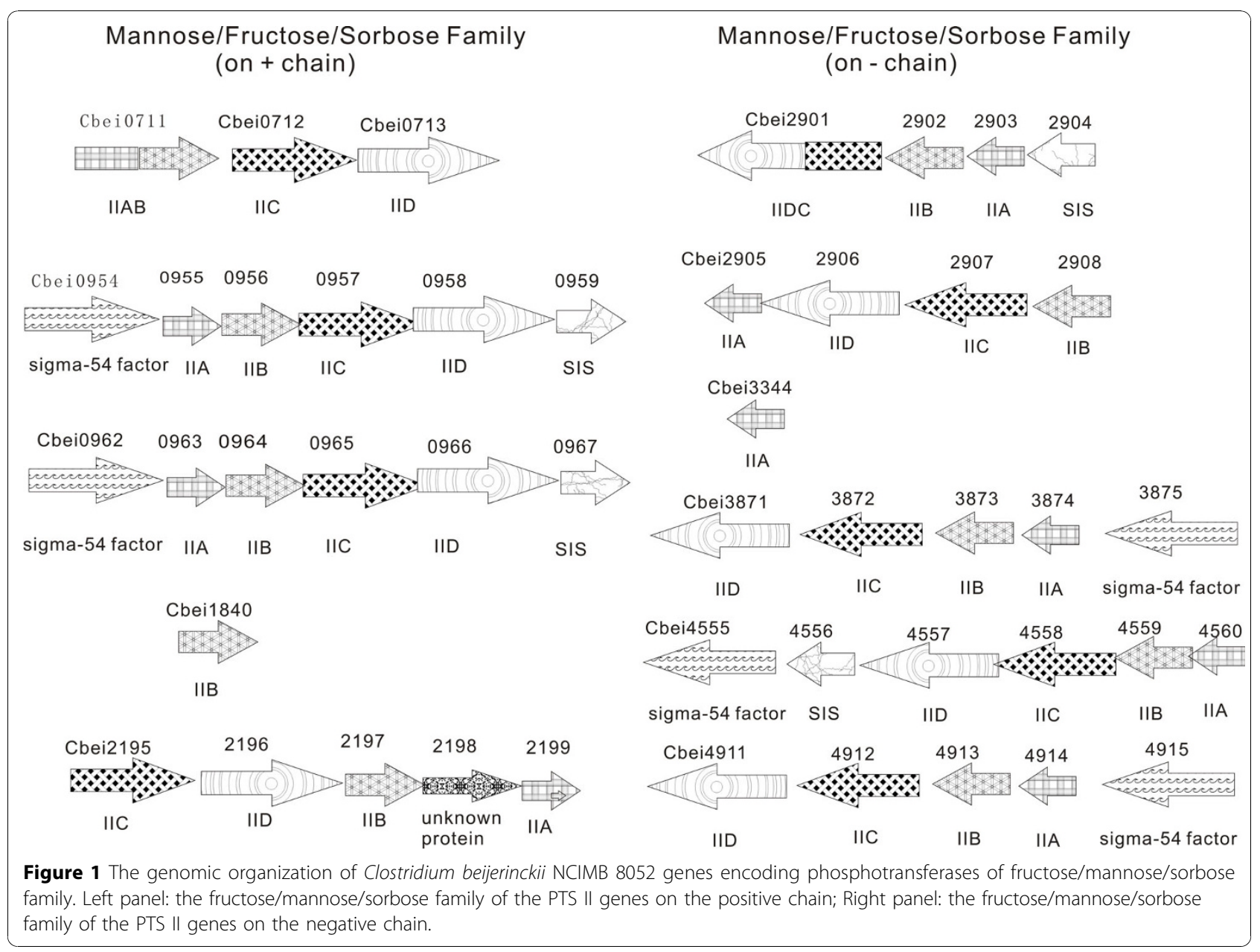

transportation and phosphorylation in bacteria [5-7]. This system is consisted of enzyme I, phosphocarrier protein (Hpr) and enzyme II complex. Enzyme I and Hpr will phosphorylate enzyme II, which is also called permease since it is the unit which carries out the transmembrane transportation. Enzyme II has A, B, C, $D$ subunits. In some microbial species these subunits each exist as a separate peptide, but in some other cases they may fuse together in various combinations. The IIB subunit is the one which decides the substrate specificity. Enzyme II can be classified into at least 7 families [8], according to their specific sugar substrates, and in different families the complex may be organized in different ways [5]. Among them, the fructose/mannose/sorbose family is the only one which has IID subunit, and all the members in this family are capable of transport fructose [6]. The most well known genes in this family, include fructose-specific ones in Bacillus subtilis [9], mannose-specific genes in Escherichia coli [10] and sorbose-specific genes in Klebsiella pneumoniae [11].
Previous studies have revealed that enzyme I and the phosphocarrier protein $(\mathrm{Hpr})$ of PTS generally has only one or two copies in clostridium strains, and they exist in the genomes monocistronically [12]. However, the genes consisted of the enzyme II complex may have multiple copies, and often form operons together with their transcription regulatory units and the genes with related catalytical functions [13]. Furthermore, the phosphotransferase systems in different types of microbial species have different mechanisms for regulation. For low GC Gram-positive bacteria like clostridium, $\mathrm{Hpr}$ is acting as an important switch. When carbon source is in short supply, $\mathrm{Hpr}$ will be phosphorylated on the serine- 46 residue by certain unique $\mathrm{Hpr}$ kinase/phosphatase. In contrast, if carbon source is sufficient, the His-15 site of $\mathrm{Hpr}$ is phosphorylated, and the PTSdependent sugar transportation and substrate-specific regulation are activated [14].

Although the study on phosphotransferase system has been going on for several decades, most of the previous reports are concentrated on limited number of model 
strains such as Escherichia coli and Bacillus subtilis. Since the beginning of this century, there are some researches on PTS in clostridium, mostly on Clostridium acetobutylicum ATCC 824, which is the first clostridium sequenced. The importance of PTS to the life process of various clostridium strains, and how the system is evolved, remain largely unknown. This work starts from the genomic data of Clostridium beijerinckii NCIMB 8052, and other sequenced clostridium strains as well as some important related model species, and analyzes the complexity of the phosphotransferase system, especially focusing on the fructose/mannose/sorbose family. We hope to explain why and how Clostridium beijerinckii NCIMB 8052 can utilize more types of sugar substrate, and propose an evolutionary model for the phosphotransferase system. It may help to illustrate the solventproducing mechanism in Clostridium beijerinckii and other related industrial bacteria, which could have theoretical and practical importance for guiding to higher butanol production through certain optimizations.

\section{Results and discussion}

Large number of PTS II genes are identified in the Clostridium beijerinckii NCIMB 8052 genome

Our study finds that there are 47 sets of PTS II genes in the Clostridium beijerinckii NCIMB 8052 genome (See Additional File 1, Table S1). That is far more than the majority of the other sequenced clostridium strains, including Clostridium botulinum A str. ATCC 3502 [12], Clostridium acetobutylicum ATCC 824 (Table S2), Clostridium perfringens strain 13 [15] (Table S3), Clostridium tetani E88 [16] (Table S4). Only Clostridium difficile 630 [17] (Table S5) has a similar number. The PTS II gene copies in other species which are evolutionarily closer to clostridium are also significantly lower than that of Clostridium beijerinckii NCIMB 8052 genome. For example, Bacillus subtilis (Table S6) has 12 sets. These findings are summarized in Table 1. There are 9 complete sets of PTS II genes which are belonging to the fructose/mannose/sorbose family among the 47 ones found in the Clostridium beijerinckii NCIMB 8052

Table 1 The bacterial strains studied and the sets of the PTS II genes in their genomes

\begin{tabular}{ll}
\hline Bacteria/strain & Sets of PTS II genes \\
\hline Clostridium beijerinckii NCIMB 8052 & 47 \\
Clostridium botulinum A str. ATCC 3502 & 15 \\
Clostridium acetobutylicum ATCC 824 & 14 \\
Clostridium perfringens strain 13 & 14 \\
Clostridium tetani E88 & 2 \\
Clostridium difficile 630 & 49 \\
Bacillus subtilis subsp. subtilis str. 168 & 12 \\
Escherichia coli str. K-12 substr. DH10B & 25 \\
\hline
\end{tabular}

Table 2 The GC distribution of the fructose/mannose/ sorbose PTS II gene sets in Clostridium beijerinckii NCIMB 8052. Three groups are indicated, with *, \#, or nothing at all

\begin{tabular}{|c|c|c|}
\hline Cbei Number & Domain Structure & GC\% \\
\hline $0711 / 0712 / 0713$ & $\|A B /\| C / \| D$ & 37.5 \\
\hline 0955/0956/0957/0958* & $\|\mathrm{A} /\| \mathrm{B} /\|\mathrm{C} /\| \mathrm{D}$ & 31.8 \\
\hline 0963/0964/0965/0966* & $\|\mathrm{A} /\| \mathrm{B} / \| \mathrm{C} / \mathrm{ID}$ & 31.9 \\
\hline 1840\# & $\| \mathrm{B}$ & 30.8 \\
\hline 2195/2196/2197/2199* & $\|C /\| D /\|B /\| A$ & 33.9 \\
\hline $2901 / 2902 / 2903^{*}$ & $\|C D /\| B / \| A$ & 32.4 \\
\hline 2905/2906/2907/2908* & $\|\mathrm{A} / \mathrm{II} /\| \mathrm{C} / \| \mathrm{B}$ & 32.0 \\
\hline $3344^{*}$ & $\| A$ & 31.7 \\
\hline 3871/3872/3873/3874\# & $\|\mathrm{D} /\| \mathrm{C} /\|\mathrm{B} /\| \mathrm{A}$ & 35.1 \\
\hline $4557 / 4558 / 4559 / 4560^{*}$ & $\|\mathrm{D} /\| \mathrm{C} /\|\mathrm{B} /\| \mathrm{A}$ & 30.3 \\
\hline 4911/4912/4913/4914\# & $\|\mathrm{D} /\| \mathrm{C} /\|\mathrm{B} /\| \mathrm{A}$ & 35.1 \\
\hline
\end{tabular}

genome, plus one additional IIA and IIB genes each (Table 2). Such a large number is never reported in any other known microbial species, including Clostridium difficile 630 (additional file 1). Their genomic organizations are illustrated in Figure 1. Interestingly, all the fructose/ mannose/sorbose family PTS II genes on the positive chain are located on the first half of the genome, while the ones on the negative chain are on the second half.

In contrast, the PTS enzyme I and the phosphocarrier protein Hpr, each have only one copy in the Clostridium beijerinckii NCIMB 8052 genome (Cbei0196 and Cbei1219, respectively). This is consistent with other clostridium species.

\section{Analysis of GC content}

To study these sets of fructose/mannose/sorbose family PTS II genes in Clostridium beijerinckii NCIMB 8052 in detail, we first calculated their GC percentages (Table 2).

As we can see from the result, the GC contents of these sets of fructose/mannose/sorbose family PTS II genes can be divided into three groups, which are indicated in the Table 2 with three different denotations, with *, \# or nothing at all.

For the two sets of Cbei3871/3872/3873/3874 and Cbei4911/4912/4913/4914, both their GC percentages are $35.1 \%$. The GC\% of Cbei0711/0712/0713 is over $37 \%$. For the remaining sets, their GC percentages are ranging from $30.3 \%$ to $33.1 \%$. Although that of Cbei2195/2196/2197/2199 is $33.9 \%$, but if the inserted hypothetical protein Cbei2198 is considered altogether, it drops to $33.1 \%$. The two orphan genes of this family, Cbei1840 and Cbei3344, are not necessarily conforming to this rule. It is noteworthy that the GC percentage of the Clostridium beijerinckii NCIMB 8052 whole genome is only $29.9 \%$, lower than that of any PTS II genes sets. We will discuss this later. 


\section{Synteny analysis}

According to Figure 1, the fructose/mannose/sorbose family PTS II genes in Clostridium beijerinckii NCIMB 8052 can also be classified into three groups based on their synteny features. One group has both sigma-54 factor and sugar isomerase (SIS) in the neighborhood, such as Cbei0955/0956/0957/0958, Cbei0963/0964/ 0965/0966 and Cbei4557/4558/4559/4560. Another group has sigma-54 factor only, including Cbei3871/ 3872/3873/3874 and Cbei4911/4912/4913/4914. The last group has neither sigma-54 factor nor sugar isomerase, like Cbei0711/0712/0713. We found these groupings are quite consistent with the ones discussed in the last section which are based on their GC percentages.

Sigma-54 is a kind of transcriptional factors, commonly existed in bacteria. It can interact with promoters to activate the transcription process. The first sigma-54 factor, rpoN, was found in 1985 through the sequencing of Klebsiella pneumoniae [18]. According to previous report [19] , in Listeria monocytogenes, sigma-54 factordependent fructose/mannose/sorbose PTS II genes are found to be associated with the bacteria's sensitivity of cytotoxin. Therefore, it is reasonable to suggest that their presence around the fructose/mannose/sorbose PTS II genes in Clostridium beijerinckii NCIMB 8052, could also have certain functional importance.

\section{Construction of phylogenetic tree}

Since IIB is the subunit which decides the PTS substrate specificity, in this part of study we use Cbei3873 as the query for blastp, and analyze the evolutionary relationship among the PTS fructose/mannose/sorbose family IIB genes in Clostridium beijerinckii NCIMB 8052 and their homologous genes identified from other representative bacteria species (selecting according to the e-value so that every e-value intervals have representations).

As we can see from the phylogenetic tree in Figure 2, the PTS fructose/mannose/sorbose family IIB genes in

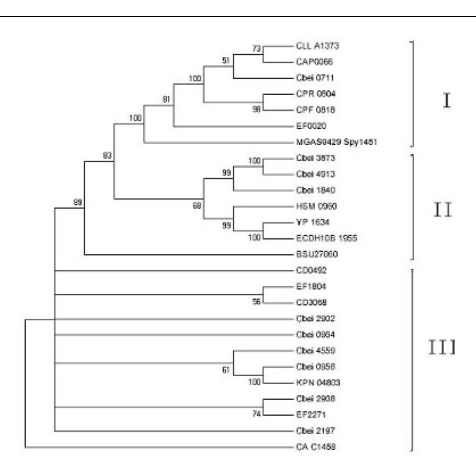

Figure 2 The phylogenetic tree built using the PTS IIB genes of fructose/mannose/sorbose family in Clostridium beijerinckii NCIMB 8052 and some representative homologs found in other bacteria.
Clostridium beijerinckii NCIMB 8052 can be grouped into three clusters too. One cluster contains Cbei0711, another includes Cbei1840, Cbei3873 and Cbei4913, while Cbei0956, Cbei0964, Cbei2902 and Cbei4559 are together in the other. Especially noteworthy is that the third cluster includes the known sorbose-specific IIB gene KPN_04803 from Klebsiella pneumoniae.

Among the group which contains Cbei1840, Cbei3873 and Cbei4913, Cbei3873 is $95 \%$ identical to Cbei4913, $75 \%$ identical to Cbei1840, and 39\% identical to the fructose-specific IIB gene Bsu27060 in Bacillus subtilis over 160 aa (68\% positive) (Figure 2). However, Cbei3873 has also been found to be $59 \%$ identical to the mannose-specific IIAB gene Ecoli_UTI89_C2014. On the other hand, although Cbei0711 is the closest to Ecoli_UTI89_C2014 in terms of gene organization since in both cases the IIA and IIB subunits are fused together, they are not grouped in the same cluster.

This result reveals the complexity of the PTS fructose/mannose/sorbose family. The reason that the transport systems for these three sugars can be classified into one family is because they are close to each other, highly homologous to each other, with few homologies to the genes in other families. But looking into it in more detail, we can find that PTS II genes for fructose and mannose transportation are closer than their relation with that of sorbose. As the result, both fructose and mannose PTS II genes phosphorylate fructose into phospho-6-fructose (F-6-P), while sorbose PTS II genes turns it into F-1-P. Furthermore, according to reference [5], the phosphorylation site on the fructose by the fructose-specific PTS genes is O-6, instead of the more common $\mathrm{O}-1$ site. This is another proof that fructose-specific PTS genes are closer to the mannose-specific ones.

\section{An evolutionary model is proposed}

To get a clearer picture of why there are so many copies of fructose/mannose/sorbose family PTS II genes in the Clostridium beijerinckii NCIMB 8052 genome, we applied the same analytical approaches to the model strain of Bacillus subtilis, B. subtilis subsp. subtilis str. 168 , by identifying all the PTS II genes in its genome, annotating and classifying each sets as well as calculating their GC percentages. The results are in Table S6 and showed that there are 12 sets of PTS II genes in B. subtilis subsp. subtilis str. 168. One of them belongs to the fructose/mannose/sorbose family, namely Bsu27040/27050/27050/27070, which phosphorylates fructose into F-6-P. The GC percentages of these PTS II gene sets in B. subtilis subsp. subtilis str. 168 are ranging from $44.4 \%$ to $50.8 \%$, with that of Bsu27040/27050/ $27050 / 27070$ is $44.4 \%$, slightly higher than the GC percentage of the whole genome, which is $43.5 \%$. 
We did the same analysis to the model strain of E. coli, Escherichia coli str. K-12 substr. DH10B (Table S7). 25 sets of PTS II genes are identified, including one complete set of the fructose/mannose/sorbose family, i.e., ECDH10B 1955/1956/1957. Their GC contents are as high as $56.1 \%$, and as low as $47.3 \%$, while that of ECDH10B 1955/1956/1957 is $51.6 \%$, close to the average GC percentage of the Escherichia coli str. K-12 substr. DH10B genome, 50.8\%.

As the results indicate, in all these bacterial species we studied, the GC percentages of PTS II gene sets are generally higher than the overall GC values of the host genomes. This leads us to speculate that the phosphotransferase system may have originated from certain high GC percentage microbial species, and during the evolutionary process lower GC percentage species acquired the components of this system from higher GC percentage species through horizontal gene transfer (HGT). The transfer may occur at an earlier stage of the evolution, since it becomes clear that the GC percentages of PTS II gene sets are being drawing closer to the average values of the host species after the transfer. For example, in Escherichia coli str. K-12 substr. DH10B, they are generally between $50-60 \%$. The values drop to the interval of $45-50 \%$ in B. subtilis subsp. subtilis str. 168. In the clostridium strains in which GC contents are even lower, the values further decrease. In the case of Clostridium beijerinckii NCIMB 8052, they are 30 to $38 \%$. Of course, this makes sense. Understandably, the sugar transport system is of the utmost importance to bacteria, concerning the life or death of each organism. So this system must have existed in microbial species for quite a long time. However, as two strains highly representative of the fructose/mannose/sorbose family, both the fructose-specific PTS II genes in Bacillus subtilis, and the mannose-specific ones in E. coli, their GC percentages are closer to the overall GC contents of their genomes. Therefore, they may be 'native' to these species and later on transferred into Clostridium beijerinckii NCIMB 8052.

To test our hypothesis that lower GC content microbial species obtained PTS II genes from higher GC ones during the evolutionary process through horizontal gene transfer, we introduce PTS II genes from the fructose/Mannitol [20] family as references (use Cbei1844 as the query for blastp, since it has been confirmed as a member). This family, which contains no IID subunit, can phosphorylate fructose into F-1-P. Like the case of Cbei1844, in a lot of species, the IIABC subunits are fused together as one gene. Since there is no IID subunit for this fructose/mannitol family, we use representative IIABC genes from the fructose/mannose/sorbose and fructose/mannitol families, run blastp separately, and use selected (selecting according to the e-value so that every e-value intervals have representations) resulting genes from both to construct another phylogenetic tree (Figure 3A). We also construct the 16sRNA tree of the species involved (Figure 3B). As shown in the 16sRNA tree, these studied species fall into two groups. Escherichia coli UTI89, Klebsiella pneumoniae subsp. pneumoniae MGH 78578 (KPN), Yersinia pestis CO92 (YPO), Yersinia pestis biovar Microtus str. 91001 (YP) and Actinobacillus succinogenes 130Z (ASuc) are grouped together (lower half of Fig. 3B). They are all Gram-negative strains with relatively higher GC contents. Another group contains all kinds of clostridium, Bacillus subtilis subsp. subtilis str. 168, Staphylococcus epidermidis ATCC 12228 (SE), Streptococcus suis 98HAH33 (SSU), Streptomyces coelicolor A3(2) (SCO), Listeria monocytogenes str. 4b F2365 (LMof) and Lactobacillus salivarius (LSL), which are all Gram-positive strains. The phylogenetic tree in Fig. 3A also includes two main branches. The upper one is the PTS II genes from the fructose/mannose/ sorbose family, while genes from the fructose/mannitol family are in the lower half. Comparing the trees in
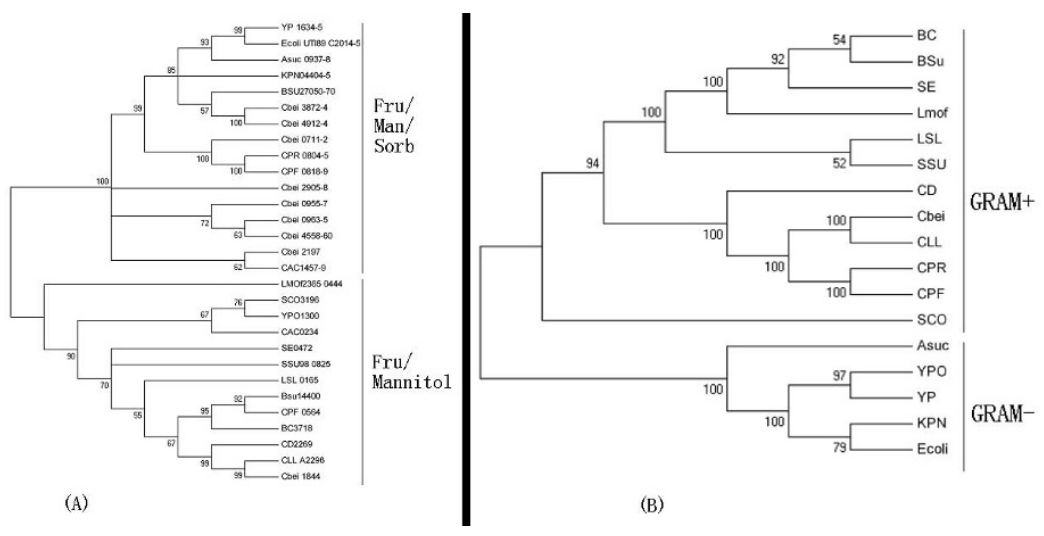

(B)

Figure 3 The Evolutionary model of the microbial PTS II system is proposed. (A) the phylogenetic tree built by using fructose/mannose/sorbose and fructose/mannitol families of the PTS II ABC genes; (B) the 16sRNA tree of the studied microbial species. 
Figs. 3A and 3B, we can find that the PTS II genes from Bacillus subtilis and clostridium strains are grouped together with those from the Gram-negative strains which we identified as the lower half in Fig. 3B. This supports our hypothesis.

\section{How the PTS II systems are evolved in Clostridium beijerinckii NCIMB 8052?}

The genome size of Clostridium beijerinckii NCIMB 8052 is about $5.9 \mathrm{MB}$, including 5104 ORFs. That is significantly larger than that of Clostridium acetobutylicum ATCC 824, which has a chromosome of $3.94 \mathrm{MB}$ plus a plasmid of 192KB [2], totaling 3917 ORFs (3739 on the chromosome and 178 on the plasmid pSol1). This is likely due to more diverse living environments enjoyed by Clostridium beijerinckii NCIMB 8052, which means more types of carbon sources it can utilize $[1,3]$. Although they are in the same genus of clostridium, they separated relatively early in the evolutionary process [21]. Clostridium acetobutylicum ATCC 824 has been shown acquiring large quantities of outside genes through horizontal gene transfer [2]; Clostridium difficile 630 also has been found with as high as $11 \%$ of mobile gene contents in its genome [17], which strongly indicates that it gained genes from other species. So, it is reasonable to expect similar events happened in C. beijerinckii NCIMB 8052 too. But in its distintive evolutionary process, C. beijerinckii NCIMB 8052 is able to keep large number of genes transferred from other species because it needs these genes to adapt to various soil environments. On the other hand, the living condition of C. acetobutylicum ATCC 824 is relatively simpler, so only fewer genes which are essential to its life activity are kept. More specifically, C. beijerinckii NCIMB 8052 is quite different from C. acetobutylicum ATCC 824 in terms of sugar needed. While C. acetobutylicum ATCC 824 is starch-based, C. beijerinckii NCIMB 8052 is molasses-based. Molasses contains $35 \%$ of sucrose, $7 \%$ of fructose and $5 \%$ of glucose (W. Jiang, personal communication). Also, C. beijerinckii NCIMB 8052 can ferment xylose degraded from agricultural wastes [22]. Since C. beijerinckii NCIMB 8052 can utilize more sugar substrates than C. acetobutylicum ATCC 824, it makes senses that it needs more genes to break down these substrates. After querying the ISFinder database (http://www-is.biotoul.fr/), we identified 92 different types of insertion sequences in the $C$. beijerinckii NCIMB 8052 genome, among them 19 with e-score lower than 1e-5 (data not shown). A lot of these insertion sequences have multiple copies. For example, ISCb1 [23] has 9 copies. In Clostridium perfringens, some PTS genes are found to be flanked by insertion sequences [24]. In C. beijerinckii NCIMB 8052, we have similar findings too. This is in agreement with our hypothesis that $C$. beijerinckii NCIMB 8052 genome gained and kept large quantities of outside genes through HGT, including some PTS genes.

Another reason which leads to the fact that there are so many sets of the PTS II genes in the C. beijerinckii NCIMB 8052 genome is gene duplication. As shown in Figure 2, many PTS IIB genes have several close-related copies. For example, Cbei3873 and Cbei4913, they are 95\% identical. Figure 1 shows that they share the same genetic organization and gene order. This strongly indicates that these genes are of great importance to the host bacteria, so they are duplicated in group, as functional backups for the original ones.

\section{The correlation between sugar utilization and PTS II gene numbers}

Clostridium beijerinckii NCIMB 8052 can better utilize fructose in the fermentation media than Clostridium acetobutylicum ATCC 824 (W. Jiang, personal communication). Our findings have shown that in the Clostridium beijerinckii NCIMB 8052 genome there are so many more gene sets of PTS II fructose/mannose/sorbose family than C. acetobutylicum ATCC 824. And with the consensus from previous reports [6] that all gene sets of this family can transport fructose, we established a likely correlation between these facts.

In addition to the PTS system, fructokinase [25] can also phosphorylate fructose into phospho-6-fructose. However, fructokinase may not have played a major role in determining the difference between the fructose utilization efficiencies of these two clostridium model strains. In both genomes, there are two copies of fructokinase genes, Cbei3497 and Cbei5009 ;CAC0424 and CAC1523.

It remains an unsolved question why these three sugars, fructose, mannose and sorbose, can form such a distinctive phosphotransferase family. In terms of their chemical structures, all three are hexose, but fructose and sorbose are ketoses while mannose is an aldose. Probably they have very similar 3D structures. The presence of sugar isomerases around certain types of the fructose/mannose/sorbose family PTS II genes in the C. beijerinckii NCIMB 8052 genome seems to suggest this too.

\section{Conclusions}

In summation of the analysis performed in this research, we found that the fructose/mannose/sorbose family of PTS II genes in Clostridium beijerinckii NCIMB 8052 can be further classified into three groups, possibly corresponding to the three different sugar substrates of this family. In addition to their respective specific sugars, all of them are capable of transport fructose. It is highly likely that this finding offers a valid explanation that 
Clostridium beijerinckii NCIMB 8052 can better utilize fructose as the sole carbon source than Clostridium acetobutylicum ATCC 824.

We also proposed an evolutionary model which suggests the horizontal gene transfer of the phosphotransferase system from higher GC microbial to lower GC ones. This may help to illustrate the mechanism why bacteria can survive in different environments through their variable carbon sources.

\section{Methods}

\section{Homology search}

The amino acid sequences of the studied genes are used as query, run blastp search [26] against the non-redundant (nr) database ,keep all the default parameters values, and find their homologous genes in other sequenced microbial species. E-value should be less than $1 \mathrm{e}-5$.

\section{Annotation of the PTS II genes}

All three methods listed below are used for the accurate annotation of the genes in the Clostridium beijerinckii NCIMB 8052 genome and other studied genomes, which are downloaded from the NCBI (http://www.ncbi. nlm.nih.gov/) RefSeq database.

(1) Blastp: described above.

(2) InterproScan [27] domain analysis: the amino acid sequences are submitted to the EBI website of InterProScan (http://www.ebi.ac.uk/Tools/InterProScan/), using all the default parameter values.

(3) The domain structures of the PTS II genes are compared with those known variations of all the families from the literatures.

\section{Synteny analysis}

For each sets of the PTS II genes, we first visually inspect their downstream and upstream regions for possible existence of synteny. The amino acid sequences of the 4 surrounding genes in both directions are picked out to identify their domains through InterproScan. They are used as the blastp queries to identify their homologous genes in other microbial species (e-value less than 1e-5). We then check the locations of these homologous genes in their respective genomes to see whether they also appear in the surrounding areas of PTS II genes.

\section{Multi-sequence alignment and construction of phylogenetic trees}

We use the embedded ClustalW [28] function of software package MEGA4 [29] for multisequence alignment, using all default parameter values. NJ (Neighbor-Joining) method [30] is applied to construct phylogenetic trees, with bootstrap values [31] calculated by repeating 500 times to evaluate the robustness of the nodes on the trees.

Additional file 1: We surveyed all the PTS genes in 7 species and listed their domain structure, predicted families as well as the GC percentage for each sets.

\section{Acknowledgements}

We would like to thank Professor Weihong Jiang and Mr. Cong Ren of the Institute of Plant Physiology \& Ecology, Shanghai Institute for Biological Sciences, Chinese Academy of Sciences for their support and useful discussions. Also, we thank colleagues including Drs. Guohui Ding, Zhiqiang Ye, Hong Sun, Mr. Hong Yu, Mr. Xiao Chang, Dr. Fudong Yu, Mr. Rudong Li and Ms. Hui Yu for their help during the study. This work was supported by National High-Tech R\&D Program (863) (2007AA02Z331).

This article has been published as part of BMC Bioinformatics Volume 11 Supplement 11, 2010: Proceedings of the 21st International Conference on Genome Informatics (GIW2010). The full contents of the supplement are available online at http://www.biomedcentral.com/1471-2105/11?issue=S11.

\section{Author details}

'Bioinformatics Center, Key Laboratory of Systems Biology, Shanghai Institutes for Biological Sciences, Chinese Academy of Sciences, Shanghai 200031, P.R. China. ${ }^{2}$ Shanghai Center for Bioinformation Technology, Shanghai 200235, P.R. China.

\section{Authors' contributions}

YS designed the study and performed the analyses and interpret the results. YS and YYL drafted the manuscript. YXL supervised the study.

\section{Competing interests}

The authors declare that they have no competing interests.

Published: 14 December 2010

\section{References}

1. Qureshi N, Saha BC, Cotta MA: Butanol production from wheat straw hydrolysate using Clostridium beijerinckii. Bioprocess Biosyst Eng 2007.

2. Nolling J, Breton G, Omelchenko MV, Makarova KS, Zeng Q, Gibson R, Lee HM, Dubois J, Qiu D, Hitti J, et al: Genome sequence and comparative analysis of the solvent-producing bacterium Clostridium acetobutylicum. J Bacteriol 2001, 183(16):4823-4838.

3. Ezeji T, Qureshi N, Blaschek HP: Butanol production from agricultural residues: Impact of degradation products on Clostridium beijerinckii growth and butanol fermentation. Biotechnology and bioengineering 2007, 97(6):1460-1469.

4. Shinto H, Tashiro $Y$, Yamashita M, Kobayashi G, Sekiguchi T, Hanai T, Kuriya $\mathrm{Y}$, Okamoto $\mathrm{M}$, Sonomoto $\mathrm{K}$ : Kinetic modeling and sensitivity analysis of acetone-butanol-ethanol production. J Biotechnol 2007, 131(1):45-56.

5. Saier MH Jr., Reizer J: Proposed uniform nomenclature for the proteins and protein domains of the bacterial phosphoenolpyruvate: sugar phosphotransferase system. J Bacteriol 1992, 174(5):1433-1438.

6. Postma PW, Lengeler JW, Jacobson GR: Phosphoenolpyruvate: carbohydrate phosphotransferase systems of bacteria. Microbiol Rev 1993, 57(3):543-594

7. Tangney M, Rousse C, Yazdanian M, Mitchell WJ: Note: sucrose transport and metabolism in Clostridium beijerinckii NCIMB 8052. J Appl Microbiol 1998, 84(5):914-919.

8. Barabote RD, Saier MH Jr.: Comparative genomic analyses of the bacterial phosphotransferase system. Microbiol Mol Biol Rev 2005, 69(4):608-634.

9. Martin-Verstraete I, Debarbouille M, Klier A, Rapoport G: Levanase operon of Bacillus subtilis includes a fructose-specific phosphotransferase system regulating the expression of the operon. J Mol Biol 1990, 214(3):657-671.

10. Erni B, Zanolari B, Kocher HP: The mannose permease of Escherichia coli consists of three different proteins. Amino acid sequence and function 
in sugar transport, sugar phosphorylation, and penetration of phage lambda DNA. J Biol Chem 1987, 262(11):5238-5247.

11. Orriss GL, Erni B, Schirmer T: Crystal structure of the IIB(Sor) domain of the sorbose permease from Klebsiella pneumoniae solved to $1.75 \mathrm{~A}$ resolution. J Mol Biol 2003, 327(5):1111-1119.

12. Mitchell WJ, Tewatia P, Meaden PG: Genomic analysis of the phosphotransferase system in Clostridium botulinum. J Mol Microbiol Biotechnol 2007, 12(1-2):33-42.

13. Yu Y, Tangney M, Aass HC, Mitchell WJ: Analysis of the Mechanism and Regulation of Lactose Transport and Metabolism in Clostridium acetobutylicum ATCC 824. Applied and environmental microbiology 2007, 73(6):1842-1850

14. Titgemeyer F, Amon J, Parche S, Mahfoud M, Bail J, Schlicht M, Rehm N, Hillmann D, Stephan J, Walter B, et al: A genomic view of sugar transport in Mycobacterium smegmatis and Mycobacterium tuberculosis. J Bacteriol 2007, 189(16):5903-5915.

15. Shimizu T, Ohtani K, Hirakawa H, Ohshima K, Yamashita A, Shiba T, Ogasawara N, Hattori M, Kuhara S, Hayashi H: Complete genome sequence of Clostridium perfringens, an anaerobic flesh-eater. Proc Natl Acad Sci U S A 2002, 99(2):996-1001.

16. Bruggemann H, Baumer S, Fricke WF, Wiezer A, Liesegang H, Decker I, Herzberg C, Martinez-Arias R, Merkl R, Henne A, et al: The genome sequence of Clostridium tetani, the causative agent of tetanus disease. Proc Natl Acad Sci U S A 2003, 100(3):1316-1321.

17. Sebaihia M, Wren BW, Mullany P, Fairweather NF, Minton N, Stabler R, Thomson NR, Roberts AP, Cerdeno-Tarraga AM, Wang H, et al: The multidrug-resistant human pathogen Clostridium difficile has a highly mobile, mosaic genome. Nat Genet 2006, 38(7):779-786.

18. Saier MH Jr., Reizer J: The bacterial phosphotransferase system: new frontiers 30 years later. Mol Microbiol 1994, 13(5):755-764.

19. Dalet K, Cenatiempo Y, Cossart P, Hechard Y: A sigma(54)-dependent PTS permease of the mannose family is responsible for sensitivity of Listeria monocytogenes to mesentericin Y105. Microbiology 2001, 147(Pt 12):3263-3269

20. Behrens S, Mitchell W, Bahl H: Molecular analysis of the mannitol operon of Clostridium acetobutylicum encoding a phosphotransferase system and a putative PTS-modulated regulator. Microbiology 2001, 147(Pt 1):75-86.

21. Keis S, Bennett CF, Ward VK, Jones DT: Taxonomy and phylogeny of industrial solvent-producing clostridia. Int I Syst Bacteriol 1995, 45(4):693-705.

22. Qureshi N, Ezeji TC, Ebener J, Dien BS, Cotta MA, Blaschek HP: Butanol production by Clostridium beijerinckii. Part I: use of acid and enzyme hydrolyzed corn fiber. Bioresour Technol 2008, 99(13):5915-5922.

23. Liyanage $H$, Holcroft $P$, Evans VJ, Keis $S$, Wilkinson SR, Kashket ER, Young M: A new insertion sequence, ISCb1, from Clostridium beijernickii NCIMB 8052. J Mol Microbiol Biotechnol 2000, 2(1):107-113.

24. Myers GS, Rasko DA, Cheung JK, Ravel J, Seshadri R, DeBoy RT, Ren Q, Varga J, Awad MM, Brinkac LM, et al: Skewed genomic variability in strains of the toxigenic bacterial pathogen, Clostridium perfringens. Genome Res 2006, 16(8):1031-1040.

25. Belouski E, Watson DE, Bennett GN: Cloning, sequence, and expression of the phosphofructokinase gene of Clostridium acetobutylicum ATCC 824 in Escherichia coli. Curr Microbiol 1998, 37(1):17-22.

26. Altschul SF, Gish W, Miller W, Myers EW, Lipman DJ: Basic local alignment search tool. J Mol Biol 1990, 215(3):403-410.

27. Zdobnov EM, Apweiler R: InterProScan-an integration platform for the signature-recognition methods in InterPro. Bioinformatics 2001, 17(9):847-848.

28. Larkin MA, Blackshields G, Brown NP, Chenna R, McGettigan PA, McWilliam H, Valentin F, Wallace IM, Wilm A, Lopez R, et al: Clustal W and Clustal X version 2.0. Bioinformatics 2007, 23(21):2947-2948.

29. Tamura K, Dudley J, Nei M, Kumar S: MEGA4: Molecular Evolutionary Genetics Analysis (MEGA) software version 4.0. Mol Biol Evol 2007. 24(8):1596-1599.

30. Saitou N, Nei M: The neighbor-joining method: a new method for reconstructing phylogenetic trees. Mol Biol Evol 1987, 4(4):406-425.

31. Efron B, Halloran E, Holmes S: Bootstrap confidence levels for phylogenetic trees. Proc Natl Acad Sci U S A 1996, 93(23):13429-13434.
doi:10.1186/1471-2105-11-S11-S9

Cite this article as: Shi et al:: Large number of phosphotransferase genes in the Clostridium beijerinckii NCIMB 8052 genome and the study on their evolution. BMC Bioinformatics 2010 11(Suppl 11):S9.

\section{Submit your next manuscript to BioMed Central and take full advantage of:}

- Convenient online submission

- Thorough peer review

- No space constraints or color figure charges

- Immediate publication on acceptance

- Inclusion in PubMed, CAS, Scopus and Google Scholar

- Research which is freely available for redistribution

Submit your manuscript at www.biomedcentral.com/submit
Biomed Central 